\title{
The Maximal Difference of Different Powers of an Element Modulo $n$
}

\author{
Jinyun Qi $(\mathbb{D})$ and Zhefeng Xu $(\mathbb{D}$ \\ School of Mathematics, Northwest University, Xi'an 710127, China \\ Correspondence should be addressed to Zhefeng Xu; zfxu@nwu.edu.cn
}

Received 31 May 2021; Accepted 30 November 2021; Published 27 December 2021

Academic Editor: Ali Jaballah

Copyright ( 2021 Jinyun Qi and Zhefeng Xu. This is an open access article distributed under the Creative Commons Attribution License, which permits unrestricted use, distribution, and reproduction in any medium, provided the original work is properly cited.

In this paper, we investigate the maximal difference of integer powers of an element modulo $n$. Let $(a)_{n}$ denote the integer $b$ with $1 \leq b \leq n$ such that $a \equiv b(\bmod n)$ for any integer $a$. Using the bounds for exponential sums, we obtain a lower bound of the function $H_{m_{1}, m_{2}}(n):=\max \left\{\left|\left(a^{m_{1}}\right)_{n}-\left(a^{m_{2}}\right)_{n}\right|: 1 \leq a \leq n,(a, n)=1\right\}$, which gives $n-H_{m_{1}, m_{2}}(n)=O\left(n^{(3 / 4)+o(1)}\right)$.

\section{Introduction}

Let $n \geq 3$ be an integer and $1 \leq a \leq n$ be an integer with $(a, n)=1$. We know that there exists a unique integer $c$ such that $a c \equiv 1(\bmod n)$, where $c$ is called the inverse of $a$ modulo $n$ with $1 \leq c<n$. We denote $c$ by $\bar{a}$. The difference between an element and its inverse modulo $n$ has been studied by several mathematicians.

Zhang [1] is the first person to explicitly study the distribution between an integer and its inverse modulo $n$, proving that

$$
\sum_{a=1}^{n}(a-\bar{a})^{2 k}=\frac{\phi(n) n^{2 k}}{(2 k+1)(k+1)}+O\left(4^{k} n^{((4 k+1) / 2)} d^{2}(n) \ln ^{2}(n)\right)
$$

where $\phi(n)$ is the Euler function and $d(n)$ is the divisor function.

Zhang [2] gives some further results for the distribution of $|a-\bar{a}|$. For any $0<\delta \leq 1$, he defines that

$$
S(n, \delta)=\#\{a: 0 \leq a \leq 1,(a, n)=1,|a-\bar{a}|<\delta n\},
$$

where $\sharp\{\triangle\}$ denotes the number of the elements in $\{\triangle\}$ and shows that

$$
S(n, \delta)=\delta(2-\delta) \phi(n)+O\left(n^{(1 / 2)} d^{2}(n) \ln ^{3}(n)\right)
$$

Meanwhile, Zheng [3] obtains similar results in a more general context. More generalizations of $[1,2]$ are in recent papers [4-7].

Our object in this paper is to study the maximal difference of different powers of an element modulo $n$. The investigation for the maximal difference of different powers of $a$ modulo $n$ is motivated by the problem of the maximal difference of $|a-\bar{a}|$, where $a$ and $\bar{a}$ run through the set $\{0,1, \ldots, n-1\}$ such that $a \bar{a} \equiv 1(\bmod n)$. Khan [8] defines

$$
M(n)=\max \{|a-\bar{a}|: 1 \leq a \leq n,(a, n)=1\},
$$

proving that

$$
M(n) \leq[n-2 \sqrt{n-1}] .
$$

Later, Khan and Shaparlinski [9] show that

$$
n-M(n)=o\left(n^{(3 / 4)+o(1)}\right) \text {. }
$$

Let $n \geq 3$ be an integer and $\mathbb{Z}_{n}$ denote residues' modulo $n$. In this paper, we assume these residues to consist of the elements $\{0,1,2, \ldots, n-1\}$. We write $\mathbb{Z}_{n}^{*}$ to be the set of elements relatively prime to $\mathbb{Z}_{n}$. Let $(a)_{n}$ be the integer $b$ with $1 \leq b \leq n$ such that $a \equiv b(\bmod n)$ for any integer $a$. Define 


$$
H_{m_{1}, m_{2}}(n):=\max \left\{\left|\left(a^{m_{1}}\right)_{n}-\left(a^{m_{2}}\right)_{n}\right|: 1 \leq a \leq n, a \in \mathbb{Z}_{n}^{*}\right\} .
$$

We prove the following theorems.

Theorem 1. Let $n \geq 3$ be an integer. Then, for arbitrary given positive integers $m_{1}, m_{2}$, we have

$$
n-H_{m_{1}, m_{2}}(n)=\mathrm{O}\left(n^{(3 / 4)+o(1)}\right) .
$$

In fact, we obtain a more general result which gives a lower bound for $H_{m_{1}, m_{2}}$ :

Theorem 2. Let $n \geq 3$ be an integer. Then, for arbitrary given positive integers $m_{1}, m_{2}$, we have

$$
H_{m_{1}, m_{2}}(n) \geq n-\frac{4\left(t^{\omega(n)} n^{(3 / 2)} d(n)+\left(\left(t^{\omega(n)} n^{(3 / 2)} d(n)\right)^{2}+\zeta(3 / 2) n^{(1 / 2)+o(1)} \sigma^{2}(n) \phi(n)\right)^{(1 / 2)}\right)}{\phi(n)}+2
$$

where $t=\max \left\{m_{1}, m_{2}\right\}$.

By applying Theorem 2, one can obtain the following.

Corollary 1. Let $n \geq 3$ be an integer. Then, for arbitrary given positive integers $m_{1}, m_{2}$, we have

$$
n-H_{m_{1}, m_{2}}(n) \leq\left(4 \zeta^{(1 / 2)}\left(\frac{3}{2}\right)+o(1)\right) n^{(9 / 4)+o(1)} \phi^{-(3 / 2)}(n) \text {. }
$$

\section{Preliminaries}

2.1. Notations. In this paper, $[t]$ is the integer part of $t$. We denote the group of units of $\mathbb{Z}_{n}$ by $\mathbb{Z}_{n}^{*}$; thus, the cardinality of $\mathbb{Z}_{n}^{*}$ is given by the Euler function $\phi(n)$. Let $\omega(n)$ be the counting function of the number of distinct primes. The number and the sum of the divisor function are defined by

$$
\begin{aligned}
& d(n)=\sum_{k \mid n} 1, \\
& \sigma(n)=\sum_{k \mid n, k>0} k=n \sum_{k \mid n, k>0} \frac{1}{k},
\end{aligned}
$$

and the Riemann zeta function is written by

$$
\zeta(s)=\sum_{z=1}^{\infty} \frac{1}{z^{s}}
$$

In what follows, we use the Landau symbol $O$ with the understanding that any implied constants are absolute. For given functions $F$ and $G$, the notation $F=O(G)$ is equivalent to the statement that the inequality $|F| \leq c|G|$ holds with some constant $c \geq 0$.

We recall the identity which follows from the formula of the sum of a geometric progression:

$$
\sum_{r=0}^{n-1} e\left(\frac{r w}{n}\right)=\left\{\begin{array}{ll}
n, & w \equiv 0(\bmod n) \\
0, & w \equiv 0(\bmod n)
\end{array},\right.
$$

where $e(z)=e^{2 \pi i z}$.
2.2. Several Lemmas. We need several lemmas to prove our theorems.

Lemma 1. For any $d \mid n$, with $d \leq n$, there holds

$$
\sum_{\substack{r \in \mathbb{Z}_{n} \\(r, n)=d}}\left|\sum_{x=0}^{h-1} e\left(\frac{r x}{n}\right)\right|^{2} \leq \frac{n h}{d}
$$

Proof. (see Lemma 17.3 in [10]).

Lemma 2. Let $p$ be a prime and $u$ be an integer with $(u, p)=1$. Then, for any integers $k \geq 2$ and $\alpha \geq 2$, there holds

$$
\left|\sum_{\substack{a=1 \\ p \nmid a}}^{p^{\alpha}} e\left(\frac{u a^{k}}{p^{\alpha}}\right)\right| \leq k p^{(\alpha / 2)}
$$

Proof. This can be easily obtained from formula (0.5) in [11].

Lemma 3. Let $n>1$ be an integer and $k$ be a fixed positive integer. For any integer $u$ with $(u, n)=1$, we have the following estimation:

$$
\left|\sum_{a \in \mathbb{Z}_{n}^{*}} e\left(\frac{u a^{k}}{n}\right)\right| \leq k^{\omega(n)} n^{(1 / 2)} .
$$

Proof. Let $n=p_{1}^{\alpha_{1}} p_{2}^{\alpha_{2}}, \ldots, p_{r}^{\alpha_{r}}$ be the prime factor decomposition of $n$. Note that

$$
\sum_{a \in \mathbb{Z}_{n}^{*}} e\left(\frac{u a^{k}}{n}\right)=\prod_{i=1}^{r}\left(\sum_{a \in \mathbb{Z}_{p_{i}^{*}}^{*}} e\left(\frac{u\left(n / p_{i}^{\alpha_{i}}\right)^{k-1} a^{k}}{p_{i}^{\alpha_{i}}}\right)\right) .
$$

In fact, if $m$ and $l$ pass through a reduced residue system modulo $n_{1}$ and $n_{2}$, respectively, $m n_{2}+\ln _{1}$ passes through a reduced system modulo $n_{1} n_{2}$. One can write 


$$
\begin{aligned}
\sum_{a \in \mathbb{Z}_{n}^{*}} e\left(\frac{u a^{k}}{n}\right) & =\sum_{m \in \mathbb{Z}_{n_{1}}^{*}} \sum_{l \in \mathbb{Z}_{n_{2}}^{*}} e\left(\frac{u\left(m n_{2}+\ln \right)^{k}}{n_{1} n_{2}}\right) \\
& =\sum_{m \in \mathbb{Z}_{n_{1}}^{*}} e\left(\frac{u\left(m n_{2}\right)^{k}}{n_{1} n_{2}}\right) \sum_{l \in \mathbb{Z}_{n_{2}}^{*}} e\left(\frac{u(\ln )^{k}}{n_{1} n_{2}}\right) \\
& =\sum_{m \in \mathbb{Z}_{n_{1}}^{*}} e\left(\frac{u n_{2}^{k-1} m^{k}}{n_{1}}\right) \sum_{l \in \mathbb{Z}_{n_{2}}^{*}} e\left(\frac{u n_{1}^{k-1} l^{k}}{n_{2}}\right) .
\end{aligned}
$$
3.

This yields identity (17). By Lemma 2, we get Lemma

Lemma 4. Let $m_{1}, m_{2}$ be fixed integers. Then, the bound,

$$
\max _{\left(u_{1}, u_{2}, n\right)=g}\left|\sum_{a \in \mathbb{Z}_{n}^{*}} e\left(\frac{u_{1} a^{m_{1}}+u_{2} a^{m_{2}}}{n}\right)\right| \leq g^{(1 / 2)} n^{(1 / 2)+o(1)},
$$

holds.

Proof. It is easily obtained from Lemma 1 in [12].

To introduce the following lemma better, we define

$$
S(d, f)=\left.\sum_{\substack{\left.r, s \in \mathbb{Z}_{n} \\ r+>0 \\ r, y\right)=d \\(s, n)=f}} \sum_{\substack{x \in \mathbb{Z}_{n}^{*} \\ \text { s. }}} e\left(\frac{r x^{m_{1}}+s x^{m_{2}}}{n}\right)|| \sum_{x=0}^{h-1} e\left(\frac{r x}{n}\right)\right|^{2}\left|\sum_{x=0}^{h-1} e\left(\frac{s x}{n}\right)\right|^{2} .
$$

Lemma 5. For any $d \mid n$ and $f \mid n$, we have

$$
S(d, f) \leq \begin{cases}m_{1}^{\omega(n)} n^{(3 / 2)} h^{3} d^{-(1 / 2)}, & f=n ; \\ m_{2}^{\omega(n)} n^{(3 / 2)} h^{3} f^{-(1 / 2)}, & d=n \\ n^{(5 / 2)} h^{2} \frac{(d, f)^{(1 / 2)}}{d f}, & d<n, f<n .\end{cases}
$$

Proof. We begin with the case when $f=n$. Apparently, this indicates that $s=0$ and $r \neq 0$. From Lemma 1 and 3 , we derive

$$
\begin{aligned}
S(d, n) & =h^{2} \sum_{\substack{r \in \mathbb{Z}_{n} \\
(r, n)=d}}\left|\sum_{x \in \mathbb{Z}_{n}^{*}} e\left(\frac{r x^{m_{1}}}{n}\right)\right|\left|\sum_{x=0}^{h-1} e\left(\frac{r x}{n}\right)\right|^{2} \\
& =h^{2} \sum_{r \in \mathbb{Z}_{n}} d\left|\sum_{x \in \mathbb{Z}_{n / d}^{*}} e\left(\frac{r / d x^{m_{1}}}{n / d}\right)\right|\left|\sum_{x=0}^{h-1} e\left(\frac{r x}{n}\right)\right|^{2} \\
& \leq n^{(r, n)=d}
\end{aligned}
$$

Using a similar method, we have the estimation,

$$
S(n, f) \leq n^{(3 / 2)} h^{3} m_{2}^{\omega(n)} f^{-(1 / 2)} .
$$

We now consider the case $d<n$ and $f<n$. By Lemma 1 and 4 , one obtains

$$
\begin{aligned}
S(d, f) \leq & n^{(1 / 2)+o(1)} \sum_{\substack{r \in \mathbb{Z}_{n} \\
(r, n)=d}} \sum_{\substack{s \in \mathbb{Z}_{n} \\
(s, n)=f}}(r, s, n)^{(1 / 2)} \\
& \left|\sum_{x=0}^{h-1} e\left(\frac{r x}{n}\right)\right|^{2}\left|\sum_{x=0}^{h-1} e\left(\frac{s x}{n}\right)\right|^{2} \leq n^{(1 / 2)+o(1)}(d, f, n)^{(1 / 2)} \\
& \frac{n^{2} h^{2}}{d f} \leq n^{(5 / 2)+o(1)} h^{2} \frac{(d, f)^{(1 / 2)}}{d f} .
\end{aligned}
$$

This completes the proof.

2.3. Proofs of the Theorems. Now, we complete the proofs of our main results.

Proof. of Theorem 2. We denote by $V$ the number of the solution of congruences:

$$
\begin{aligned}
& x^{m_{1}} \equiv h-y+z(\bmod n), \\
& x^{m_{2}} \equiv-h+u-v(\bmod n) .
\end{aligned}
$$

In the following, we show the range of $h$ in the case that congruences (25) are solvable for $x \in \mathbb{Z}_{n}^{*}$, and integers $0 \leq y, z, u, v \leq h-1$. Then, it can be easily deduced that

$$
H_{m_{1}, m_{2}}(n) \geq n-4 h+2 \text {. }
$$

Applying the exponential sums identity (13), we have

$$
\begin{aligned}
V & =\sum_{x \in \mathbb{Z}_{n}^{*}} \sum_{y, z, u, v=0}^{h-1} \frac{1}{n^{2}} \\
& \sum_{r, s \in \mathbb{Z}_{n}} e\left(\frac{r\left(x^{m_{1}}-h+y-z\right)+s\left(x^{m_{2}}+h-u+v\right)}{n}\right) \\
= & \frac{1}{n^{2}} \sum_{r, s \in \mathbb{Z}_{n}} e\left(\frac{-r h+s h}{n}\right) \sum_{x \in \mathbb{Z}_{n}^{*}} e\left(\frac{r x^{m_{1}}+s x^{m_{2}}}{n}\right) \\
& \left|\sum_{x=0}^{h-1} e\left(\frac{r x}{n}\right)\right|^{2}\left|\sum_{x=0}^{h-1} e\left(\frac{s x}{n}\right)\right|^{2} .
\end{aligned}
$$

Provided that $r=0$ and $s=0$, the right-hand side of equation (27) is $\left(\phi(n) h^{4} / n^{2}\right)$. Then, we have

$$
\begin{aligned}
\left|V-\frac{\phi(n) h^{4}}{n^{2}}\right| \leq \frac{1}{n^{2}} \sum_{\substack{r, s \in \mathbb{Z}_{n} \\
r+s>0}}\left|\sum_{x \in \mathbb{Z}_{n}^{*}} e\left(\frac{r x^{m_{1}}+s x^{m_{2}}}{n}\right)\right| \\
\left|\sum_{x=0}^{h-1} e\left(\frac{r x}{n}\right)\right|^{2}\left|\sum_{x=0}^{h-1} e\left(\frac{s x}{n}\right)\right|^{2} .
\end{aligned}
$$


From Lemma 5, one can write

$$
\begin{aligned}
\left|V-\frac{\phi(n) h^{4}}{n^{2}}\right| & \leq \frac{1}{n^{2}} \sum_{r+s>0}\left|\sum_{x \in \mathbb{Z}_{n}^{*}} e\left(\frac{r x^{m_{1}}+s x^{m_{2}}}{n}\right)\right|\left|\sum_{x=0}^{h-1} e\left(\frac{r x}{n}\right)\right|^{2}\left|\sum_{x=0}^{h-1} e\left(\frac{s x}{n}\right)\right|^{2} \\
& =\frac{1}{n^{2}}\left(\sum_{d \mid n, d<n} S(d, n)+\sum_{f \mid n, f<n} S(n, f)+\sum_{d|n, f| n} S(d, f) \mid\right. \\
& \leq \frac{1}{n^{2}}\left(\left(m_{1}^{\omega(n)}+m_{2}^{\omega(n)}\right) n^{(3 / 2)} h^{3} d(n)+n^{(5 / 2)+o(1)} h^{2} \sum_{d|n, f| n} \frac{(d, f)^{(1 / 2)}}{d f}\right) .
\end{aligned}
$$

We know

$$
\begin{aligned}
\sum_{d|n, f| n} \frac{(d, f)^{(1 / 2)}}{d f} & \leq \sum_{k \mid n} k^{(1 / 2)} \sum_{d \mid(n / k)} \sum_{f \mid(n / k)} \frac{1}{k^{2} d f} \\
& =\sum_{k \mid n} k^{-(3 / 2)} \sum_{d \mid(n / k)} \sum_{f \mid(n / k)} \frac{1}{d f} \leq \zeta\left(\frac{3}{2}\right)\left(\frac{\sigma(n)}{n}\right)^{2} .
\end{aligned}
$$

Remark that $t=\max \left\{m_{1}, m_{2}\right\}$, so we can get the inequality

$$
\begin{aligned}
\left|n^{2} V-\phi(n) h^{4}\right| \leq & \left(m_{1}^{\omega(n)}+m_{2}^{\omega(n)}\right) n^{(3 / 2)} h^{3} d(n) \\
& +\zeta\left(\frac{3}{2}\right) \sigma^{2}(n) n^{(1 / 2)+o(1)} h^{2} \leq 2 t^{\omega(n)} n^{(3 / 2)} h^{3} d(n) \\
& +\zeta\left(\frac{3}{2}\right) \sigma^{2}(n) n^{(1 / 2)+o(1)} h^{2}
\end{aligned}
$$

The right-hand side of (32) has to be positive. It suffices to show that

$$
\phi(n) h^{2}-2 t^{\omega(n)} n^{(3 / 2)} h d(n)-\zeta\left(\frac{3}{2}\right) \sigma^{2}(n) n^{(1 / 2)+o(1)} \geq 0 .
$$

Multiplying both side of inequality (33) by $\phi(n)$ and calculating briefly, we have

$$
\begin{aligned}
\left(\phi(n) h-n^{(3 / 2)} d(n) t^{\omega(n)}\right)^{2} \geq & \left(n^{(3 / 2)} d(n) t^{\omega(n)}\right)^{2} \\
& +\zeta\left(\frac{3}{2}\right) \sigma^{2}(n) n^{(1 / 2)+o(1)} .
\end{aligned}
$$

Hence,

After a simple calculation, one can write

$$
h \geq\left(t^{\omega(n)} n^{(3 / 2)} d(n)+\left(\left(t^{\omega(n)} n^{(3 / 2)} d(n)\right)^{2}+\zeta\left(\frac{3}{2}\right) n^{(1 / 2)+o(1)} \sigma^{2}(n) \phi(n)\right)^{(1 / 2)}\right) \phi^{-1}(n) .
$$

This finishes the proof of Theorem 2.

Proof. of Corollary 1. Applying Theorem 2, one can obtain

$$
\begin{aligned}
& n-H_{m_{1}, m_{2}}(n) \\
& \leq 4\left(t^{\omega(n)} n^{(3 / 2)} d(n)+\left(\left(t^{\omega(n)} n^{(3 / 2)} d(n)\right)^{2}+\zeta\left(\frac{3}{2}\right) n^{(1 / 2)+o(1)} \sigma^{2}(n) \phi(n)\right)^{(1 / 2)}\right) \phi^{-1}(n) \\
& \leq 4\left(2 t^{\omega(n)} n^{(3 / 2)} d(n)+\zeta^{(1 / 2)}\left(\frac{3}{2}\right) n^{(1 / 4)+o(1)} \sigma(n) \phi^{(1 / 2)}(n)\right) \phi^{-1}(n)
\end{aligned}
$$




$$
\begin{aligned}
& \leq 8 t^{\omega(n)} n^{(3 / 2)} d(n) \phi^{-1}(n)+4 \zeta^{(1 / 2)}\left(\frac{3}{2}\right) n^{(9 / 4)+o(1)} \phi^{-(3 / 2)}(n) \\
& \leq\left(4 \zeta^{(1 / 2)}\left(\frac{3}{2}\right)+o(1)\right) n^{(9 / 4)+o(1)} \phi^{-(3 / 2)}(n),
\end{aligned}
$$

where we use the bounds

$$
\begin{aligned}
\sigma(n) \phi(n) & \leq n^{2}, \\
\omega(n) & =O\left(\frac{\log n}{\log \log n}\right), \\
\log d(n) & =O\left(\frac{\log n}{\log \log n}\right) .
\end{aligned}
$$

The proof of Corollary 1 is complete.

Proof. of Theorem 1. Combining Corollary 1 with the bounds,

$$
\frac{n}{\phi(n)}=O(\log \log (n))
$$

See Theorem 328 in [10]; Theorem 1 follows immediately.

\section{Data Availability}

No data were used to support this study.

\section{Conflicts of Interest}

The authors declare that they have no conflicts of interest.

\section{Acknowledgments}

This work was supported by the Basic Research Program for Nature Science of Shaanxi Province (2014JM1001 and 2015KJXX-27) and N.S.F.(11971381, 11471258 , and $11701447)$ of P. R. China.

\section{References}

[1] W. Zhang, "On the difference between an integer and its inverse modulo n," Journal of Number Theory, vol. 52, no. 1, pp. 1-6, 1995.

[2] W. Zhang, "On the distribution of inverses modulo $n$," Journal of Number Theory, vol. 61, no. 2, pp. 301-310, 1996.

[3] Z. Zheng, "The distribution of zeros of an irreducible curve over a finite field," Journal of Number Theory, vol. 59, no. 1, pp. 106-118, 1996.

[4] C. Cobeli and A. Zaharescu, "On the distribution of the $\mathbb{F}_{p}$-points on an affine curve in $\mathrm{r}$ dimensions," Acta Arithmetica, vol. 99, no. 4, pp. 321-329, 2001.

[5] A. Granville, I. E. Shparlinski, and A. Zaharescu, "On the distribution of rational functions along a curve over and residue races," Journal of Number Theory, vol. 112, no. 2, pp. 216-237, 2005.

[6] M. Vajaitu and A. Zaharescu, "Distribution of values of rational maps on the F p -points on an affine curve," Monatshefte for Mathematik, vol. 136, no. 1, pp. 81-86, 2002.
[7] Z. Xu, "Distribution of the difference of an integer and its $\mathrm{m}$-th power mod $\mathrm{n}$ over incomplete intervals," Journal of Number Theory, vol. 133, no. 12, pp. 4200-4223, 2013.

[8] M. R. Khan, "An optimization with a modular constraint: 10736," The American Mathematical Monthly, vol. 108, no. 4, pp. 374-375, 2001.

[9] M. R. Khan and I. E. Shparlinski, "On the maximal difference between an element and its inverse modulo $n$," Periodica Mathematica Hungarica, vol. 47, no. 1-2, pp. 11-117, 2003.

[10] G. H. Hardy and E. M. Wright, An Introduction to the Theory of Numbers, The Clarendon Press, Oxford University Press, New York, NY, USA, Fifth edition, 1979.

[11] T. Cochrane and Z. Zheng, "Upper bounds on a two-term exponential sum," Science in China, Series A: Mathematics, vol. 44, no. 8, pp. 1003-1015, 2001.

[12] I. E. Shparlinski, "Modular hyperbolas," Japanese Journal of Mathematics, vol. 7, no. 2, pp. 235-294, 2012. 